Max-Planck-Institut für demografische Forschung

Max Planck Institute for Demographic Research

Konrad-Zuse-Strasse 1 - D-18057 Rostock · GERMANY

Tel +49 (0) 3812081 - 0; Fax +49 (0) 3812081 - 202;

http://www.demogr.mpg.de

MPIDR WORKING PAPER WP 2006-052

DECEMBER 2006

\title{
Travail des femmes, caractéristiques familiales et sociales: le cas du Maroc
}

\author{
Anna Paterno \\ Giuseppe Gabrielli \\ Agata V. D’Addato (daddato@demogr.mpg.de)
}

This working paper has been approved for release by: Laura Bernardi (bernardi@ demogr.mpg.de) Head of the Independent Research Group on The Culture of Reproduction.

(C) Copyright is held by the authors.

Working papers of the Max Planck Institute for Demographic Research receive only limited review. Views or opinions expressed in working papers are attributable to the authors and do not necessarily reflect those of the Institute. 


\title{
Travail des femmes, caractéristiques familiales et sociales: le cas du Maroc
}

\author{
Anna Paterno ${ }^{*}$, Giuseppe Gabrielli ${ }^{*}$, Agata V. D'Addato ${ }^{* * 1}$
}

\section{Résume:}

Le taux d'activité féminine au Maroc, malgré un trend mouvant dans les années entre la fin du XX siècle et le nouveau millénaire n'a pas subi des accroissements remarquables.

Dans ce travail nous mettons en relief quelques facteurs qui expliquent la participation féminine au marché du travail marocain. On poursuit ce but dans une optique comparative «temporelle», en observant l'évolution qui a eu lieu approximativement pendant la dernière décennie, et «spatiale», en distinguant les milieux urbains des milieux ruraux.

Dans cette optique, nous traçons en premier lieu le cadre général des caractéristiques sociales, familiales et d'emploi des femmes marocaines, faisant recours aux principales informations officielles produites sur place, malgré leur caractère fragmentaire. Dans la suite du travail, nous utilisons les données issues de deux enquêtes conduites sur le territoire national en 1992 et entre fin 2003 et début 2004, qui s'inscrivent dans le programme international «Demographic and Health Surveys»(DHS). Finalement, des analyses multivariées mettent en lumière les principaux déterminants du désir des Marocaines de faire partie du marché du travail, et les motivations les plus significatives à la base de leur insertion dans un secteur d'activité spécifique.

Les analyses effectuées mettent en évidence que la participation féminine au marché du travail marocain reste liée, surtout en milieu rural, à des modèles familiaux traditionnels dans lesquels le rôle des femmes est limité à des fonctions secondaires, souvent en dehors du travail rémunéré. Les résultats obtenus suggèrent aussi que la stabilité substantielle de la participation féminine au marché du travail observée pourrait être considérée plus que comme une «occasion perdue» dans le chemin d'une amélioration du statut des femmes, plutôt comme une étape obligée du processus de leur affranchissement des activités dégradantes d'un système de production arriéré, étape nécessaire afin d'atteindre un niveau de développement global plus élevé.

\footnotetext{
* Dipartimento per lo Studio delle Società Mediterranee, Université de Bari.

** Max Planck Institute for Demographic Research (Rostock).

${ }^{1}$ Ce travail est le fruit de la collaboration entre les auteurs; cependant, en ce qui concerne le texte, la partie 3 est à attribuer à A.V. D' Addato, la partie 4 à G. Gabrielli et la partie 2 à A. Paterno. L'introduction et les conslusions sont communes aux auteurs.
} 


\section{Introduction}

Le taux d'activité féminine au Maroc, tout en étant assez proche de ceux des autres pays de la rive sud-est du bassin méditerranéen (la Tunisie, l'Egypte, la Turquie, etc.), est beaucoup plus bas que les niveaux typiques des pays européens. Cette situation, qui est communément attribuée à la persistance de mentalités et de comportements très différents de ceux qui dominent dans des pays «occidentaux», semble être le résultat d'un ensemble de motivations politiques, sociales, culturelles et démographiques, au delà de l'évolution des années passées.

Sur la base de ces considérations, dans ce travail nous nous proposons de mettre en relief quelques facteurs qui expliquent la participation féminine au marché du travail marocain entre le début des années ' 90 et les premières années du $\mathrm{XXI}^{\mathrm{e}}$ siècle. En particulier, nous essayons de mettre en évidence, d'un côté, l'influence de l'environnement social et, de l'autre, l'impact des caractéristiques individuelles et familiales, sur l'accès féminin au travail extradomestique. On poursuit ce but dans une optique comparative «temporelle», afin de mettre en lumière l'évolution qui a eu lieu approximativement pendant la dernière décennie, et «spatiale», afin de prendre en considération les implications de la rapide urbanisation en cours dans le pays nord-africain, en distinguant les milieux urbains des milieux ruraux (de SarnoPrignano A., El Mouaatamid B., Paterno A., 2003).

Dans cette optique, nous allons tracer en premier lieu le cadre général de la situation d'emploi des femmes marocaines dans cette période, ayant recours aux principales informations officielles produites sur place, malgré les difficultés liées à leur caractère fragmentaire. Dans la suite du travail, nous utilisons les données issues de deux enquêtes conduites avec les mêmes modalités sur le territoire national en 1992 et entre fin 2003 et début 2004, qui s'inscrivent dans le programme international «Demographic and Health Surveys» (DHS). Ces données nous permettent de décrire plus en détail les caractéristiques sociales, démographiques et familiales des Marocaines qui travaillent, en comparaison avec celles qui ne travaillent pas. Finalement, des analyses multivariées mettront en lumière les principaux déterminants du désir des Marocaines observées de faire partie du marché du travail, et les motivations les plus significatives à la base de leur insertion dans un secteur d'activité spécifique (agriculture, industrie, services).

De l'ensemble de ces informations, on voit d'une part l'influence des caractéristiques individuelles sur la participation féminine au marché du travail marocain, et d'autre part des indications utiles sur le niveau général du développement de l'environnement social dans lequel les interviewées vivent et sur le niveau d'émancipation des femmes marocaines, en faisant référence aux différentes époques et aux différents contextes territoriaux observés.

\section{Le contexte de fond: caractéristiques sociales, familiales et d'emploi des femmes marocaines au niveau agrégé}

Depuis quelques décennies, le Maroc vit une profonde transition. De nombreuses transformations semblent profondément corrélées et se reflètent dans plusieurs milieux. À la lumière des objectifs de ce travail, il nous semble opportun d'examiner la portée et les principales caractéristiques de l'évolution socio- 
démographique qui a eu lieu dans le pays approximativement entre le début des années '90 et le début de la décennie suivante (1992-2003) ${ }^{2}$, dans les secteurs influençant le plus significativement le marché du travail et la participation féminine aux activités productives. Bien évidemment, ce n'est pas facile de distinguer et de diviser nettement ces secteurs, qui sont en réalité «tressés» les uns avec les autres. Il est d'autant moins facile d'en tracer l'évolution que les informations quantitatives disponibles sont fragmentaires. Cependant, cette lacune est en partie compensée par l'existence d'une littérature riche et intéressante, produite surtout sur place, dont l'examen nous permettra d'offrir un «contexte de fond» de la situation féminine au Maroc, dans les domaines juridique, politicosocial, culturel, familial et d'emploi. Une observation synthétique des conditions de vie des femmes marocaines donne l'impression que, bien que l'on ait fait beaucoup de pas vers une amélioration de leur statut, le chemin qui reste est encore plutôt tortueux. Un examen plus approfondi des différents contextes confirme cette impression.

En commençant par le milieu plus «formel», soit le milieu juridique, on remarque que, malgré les récentes dispositions législatives établissant l'égalité entre les sexes, à commencer par la réforme du code du statut personnel de 1993 et par la réforme du code de la famille de 2004, les discriminations de genre n'ont pas été complètement éliminées. Par exemple, les normes confirment que la femme doit obéir à son mari et donnent le contrôle de la famille à l'homme. Ces dispositions législatives réglementent également le droit au divorce, qui n'est donné qu'à l'homme. L'homme a aussi la possibilité de nier à sa femme le droit de travailler hors du foyer domestique. En outre, la juridiction marocaine permet toujours la polygamie, malgré le fait que récemment certaines mesures ont été prises afin de protéger la femme contre cette institution, et malgré sa diffusion de plus en plus faible. En résumé, «les pesanteurs sociales, culturelles et certaines dispositions législatives consacrent la primauté de l'homme sur la femme, engendrant ainsi les facteurs de blocage de l'égalité des sexes. Il faut admettre que la reconnaissance juridique de l'égalité entre les sexes ne peut à elle seule éliminer la discrimination de fait» (Chaouai A., 1998, page 156).

Même du point de vue politico-social, les institutions responsables de promouvoir la condition de la femme et les actions entreprises se sont souvent confrontées, d'une part, à l'insuffisance de moyens matériels et financiers nécessaires à la mise en œuvre des projets élaborés et, de l'autre, à une conception de la femme comme sujet «passif» plutôt que comme «acteur» dans le processus de développement. Globalement, donc, malgré le fait que «les lois» et «l'état» donnent aux femmes les mêmes droits qu'aux hommes, et malgré certains récents progrès vers la réalisation d'une égalité de genre, la mentalité plus traditionnelle met encore la figure féminine dans une condition d'infériorité et de dépendance par rapport à l'homme (Harrami N., 2000; Mosleh K., 2000).

Cette conception influence aussi la position de la femme dans l'éducation et la formation. En effet, les nombreuses interventions mises en place pour éliminer les obstacles à l'accès féminin dans le système scolaire n'ont pas totalement éliminé les écarts qui caractérisent les niveaux de scolarisation, surtout en milieu rural. Les

\footnotetext{
${ }^{2}$ Nous précisons que 1992 et 2003 correspondent aux années civiles dans lesquels les enquêtes utilisées dans la suite de notre travail ont été réalisées; pour cette raison nous avons choisi ces dates comme référence chronologique dans cette partie aussi.
} 
données présentées dans le tableau $1^{3}$ démontrent, en premier lieu, qu'au cours de la période observée, la proportion des filles sur le total des élèves, tout en demeurant minoritaire en comparaison avec les garçons, a constamment augmenté; cela se vérifie dans tous les ordres d'école. Également, l'effectif des filles inscrites à tous les niveaux d'éducation a subi des accroissements supérieurs à ceux de l'effectif des garçons. À l'échelle nationale et pour tous les cycles de formation, l'effectif des étudiantes pour l'année scolaire 2002-03 a été, en moyenne, environ $50 \%$ plus élevé qu'en $1992-93^{4}$. Cependant, la donnée qui mérite particulièrement notre attention concerne les progrès qui caractérisent les milieux ruraux, où la proportion de filles inscrites est passée de moins de $1 / 3$ du total, à environ 1/2. Malgré cela, dans les ordres supérieurs de l'éducation, de grands écarts persistent entre les «villes», où la proportion d'étudiantes est presque aussi grande que celle des étudiants, et la «campagne», où les valeurs enregistrées chez les filles sont environ la moitié de celles enregistrées chez les garçons. À tout cela il faut ajouter que la lutte contre l'analphabétisme a produit des résultats plus faibles chez les femmes, surtout chez les résidentes de milieu rural (Hddigui E.M., 1998).

Tableau 1 -Quelques caractéristiques sociales, démographiques et d'emploi des femmes marocaines par milieu de résidence, $1992-2003$.

\begin{tabular}{|c|c|c|c|c|c|c|c|c|c|c|}
\hline \multirow{2}{*}{ Années } & \multicolumn{2}{|c|}{$\begin{array}{c}\text { Étudiantes }^{(*)} \\
\text { (v.a.) }\end{array}$} & \multicolumn{2}{|c|}{$\begin{array}{l}\text { Étudiantes }^{(*)} \\
(\% \text { sur le total) }\end{array}$} & \multicolumn{2}{|c|}{ ISF } & \multicolumn{2}{|c|}{ Taux d'activité } & \multicolumn{2}{|c|}{$\begin{array}{l}\text { Taux de } \\
\text { chômage }\end{array}$} \\
\hline & Urbain & Rural & Urbain & Rural & Urbain & Rural & $\begin{array}{l}\text { Urba } \\
\text { in }\end{array}$ & Rural & Urbain & Rural \\
\hline 1992 & 1.219 .210 & 324.288 & 44,8 & 30,1 & $2,54^{(* *)}$ & $5,54^{(* *)}$ & 23,2 & - & 25,3 & - \\
\hline 1993 & 1.257 .204 & 385.572 & 45,2 & 31,8 & 2,56 & 4,25 & 22,1 & - & 21,7 & - \\
\hline 1994 & 1.290 .031 & 436.177 & 45,4 & 33,1 & 2,30 & 4,10 & - & - & - & - \\
\hline 1995 & 1.317 .436 & 472.554 & 45,5 & 34,0 & - & - & 30,5 & 39,5 & 32,2 & 6,5 \\
\hline 1996 & 1.334 .908 & 507.529 & 45,9 & 34,8 & - & - & 25,8 & - & 23,6 & - \\
\hline 1997 & 1.336 .727 & 565.167 & 46,3 & 36,3 & 2,30 & 4,10 & 24,0 & - & 21,8 & - \\
\hline 1998 & 1.377 .061 & 663.998 & 46,6 & 38,7 & 2,30 & 4,00 & 22,3 & - & 24,4 & - \\
\hline 1999 & 1.427 .654 & 757.793 & 46,9 & 40,5 & 2,30 & 3,90 & 22,8 & 40,9 & 27,6 & 2,1 \\
\hline 2000 & 1.480 .390 & 837.881 & 47,1 & 41,8 & 2,10 & 3,30 & 21,3 & 37,5 & 26,7 & 1,7 \\
\hline 2001 & 1.532 .141 & 924.996 & 47,4 & 42,8 & 2,10 & 3,30 & 20,1 & 33,5 & 21,0 & 1,6 \\
\hline 2002 & 1.559 .644 & 972.639 & 47,7 & 43,3 & 2,10 & 3,10 & 19,8 & 32,7 & 24,2 & 1,7 \\
\hline 2003 & 1.583 .391 & 976.272 & 47,9 & 43,4 & $2,10^{(* * *)}$ & $3,10^{(* * *)}$ & 20,9 & 37,3 & 25,8 & 1,6 \\
\hline
\end{tabular}

${ }^{(*)}$ Années scolaires de 1992-93 à 2002-03. - = Cette information n'est pas disponible.

Source: Direction de la Statistique et CERED, années variées.

${ }^{(* *)}$ Azelmat M., Ayad M., Housni E.A., 1993. ${ }^{(* * *)}$ Azelmat M., Ayad M., Abdelmoneim A., 2005.

Même en ce qui concerne le type de formation culturelle et professionnelle, la littérature marocaine confirme que la ségrégation des femmes dans des secteurs d'activité spécifiques, et généralement de bas niveau, est fortement liée à leurs choix (ou à ceux de leurs parents) dans le cadre de l'éducation, étant donné qu'elles se concentrent dans les secteurs plus traditionnels exigeant moins de qualifications (Boutata M., 2005). En général, donc, les différentiels de genre dans le niveau d'éducation sont dus à un ensemble de facteurs: d'abord la persistance d'une mentalité qui attribue, déjà dans le cadre des ménages et depuis la première enfance, différents rôles aux garçons et aux filles, et qui relègue les femmes à des

\footnotetext{
${ }^{3}$ Le choix des indicateurs sélectionnés dans cette partie a été fortement conditionné par la disponibilité des données et, en particulier, par la difficulté de construire les séries historiques correspondantes, souvent marquées par des lacunes et par l'hétérogénéité des sources.

${ }^{4}$ L'augmentation des filles inscrites - et des garçons inscrits - apparaît encore plus marquée si l'on considère que l'effectif de la population en âge scolaire entre 1992 et 2003 a baissé.
} 
positions familiales et professionnelles marquées par une générale subordination à celles des hommes. Toutefois, dans ce contexte, d'autres facteurs interviennent, comme le manque de moyens financiers à cause de l'incidence élevée de la pauvreté, la nécessité d' «utiliser» depuis l'enfance les enfants dans le travail domestique et/ou extradomestique et, surtout, la grande diffusion de mariages et de maternités précoces (CERED, 1998a).

À ce propos, il nous semble nécessaire de souligner qu'au Maroc les variations les plus significatives du comportement nuptial et fécond se sont vérifiées à partir du milieu des années '70 dans les zones urbaines et pendant les années '90 en milieu rural. Focalisant l'attention sur cette dernière décennie, on note, par exemple, que l'âge moyen des femmes au moment du mariage a augmenté surtout en milieu rural, atteignant, en 2004, 25,5 ans, contre 27,1 ans dans les villes (CERED, 2005). De la même façon, les données présentées dans le tableau 2 mettent en évidence que la proportion de femmes célibataires a fortement augmenté, et cela surtout dans les classes d'âge supérieures à 30 ans. Ces données démontrent, selon nous, que l'union matrimoniale au Maroc paraît de moins en moins un comportement précoce et universel. Notamment dans les villes, on peut entrevoir de nouvelles figures de femmes qui réservent un espace plus réduit, dans leur vie, au mariage, tout en demeurant assez loin de la typologie classique des «singles» occidentaux et tout en restant bien liées à l'institution familiale (CERED, 1998b).

Tableau 2 - Proportions de femmes célibataires par groupes d'âge.

\begin{tabular}{|c|c|c|c|c|c|c|c|c|c|}
\hline \multirow{2}{*}{$\begin{array}{l}\text { Groupe } \\
\text { d'âge }\end{array}$} & \multicolumn{9}{|c|}{ Années } \\
\hline & 1992 & 1994 & 1995 & 1997 & 1998 & 1999 & 2000 & 2001 & 2003 \\
\hline $15-19$ & 87,5 & 87,2 & 89,5 & 87,3 & 91,5 & 91,4 & 91,3 & 92,4 & 89,0 \\
\hline $20-24$ & 56,0 & 55,6 & 60,2 & 60,8 & 65,3 & 67,2 & 69,0 & 69,8 & 61,7 \\
\hline $25-29$ & 34,3 & 35,1 & 39,8 & 41,1 & 47,4 & 44,8 & 46,1 & 47,5 & 40,7 \\
\hline $30-34$ & 13,9 & 18,3 & 20,8 & 24,6 & 25,9 & 27,6 & 29,8 & 31,1 & 26,9 \\
\hline $35-39$ & 6,7 & 9,2 & 11,6 & 11,5 & 15,2 & 15,0 & 16,8 & 17,9 & 16,3 \\
\hline $40-44$ & 2,6 & 4,9 & 5,6 & 6,9 & 5,0 & 7,7 & 8,5 & 9,2 & 11,3 \\
\hline $45-49$ & 0,4 & 2,1 & 0,6 & 3,4 & 2,2 & 3,4 & 4,1 & 4,0 & 4,6 \\
\hline
\end{tabular}

Source: Direction de la Statistique, années variées.

Concernant la fécondité, les nombreuses enquêtes réalisées et la littérature très riche mettent en évidence que le comportement des Marocaines a subi, surtout en milieu urbain, des changements forts qui ont conduit à une baisse marquée de tous les indicateurs disponibles. Notamment, les taux bruts de natalité entre 1992-93 et 2002-03 ont passé de $21,2 \%$ à $19,2 \%$ en «ville» et de $33,5 \%$ à $23,3 \%$ à la «campagne» (Direction de la Statistique et CERED, années variées). Les taux de fécondité ont aussi baissé, en passant respectivement, en milieu urbain et rural, de 2,54 et 5,56 enfants par femme au tout début des années ' 90 , à 2,10 et 3,00 au début du nouveau siècle. Cette contraction de la fécondité est particulièrement forte chez les femmes de 20 à 29 ans. De plus, comme on l'a remarqué pour la nuptialité, pour la fécondité dans la période observée, une générale postposition du calendrier des naissances est enregistrée (CERED, 1997).

Faute d'espace, nous ne mentionnerons pas ici les nombreux déterminants de

\footnotetext{
${ }^{5}$ On rappelle que 2,1 est la dite «valeur de seuil» de l'ISF, soit le nombre moyen d'enfants par femme qui distingue les populations en potentielle expansion démographique des populations en potentielle diminution, et que cette valeur est typique des pays ayant déjà terminé leur transition démographique.
} 
l'évolution synthétisée jusqu'ici. Notons cependant les interventions gouvernementales adressées à la diffusion des pratiques contraceptives, le processus d'urbanisation, la raréfaction de certains modèles familiaux traditionnels (par exemple, les ménages complexes) et l'augmentation des niveaux d'éducation féminine; dans tous ces processus les progrès faits dans une dimension sont à la fois cause et effet des changements dans les autres sphères de comportement (D’Addato A.V., 2006a; 2006b).

Dans cette optique, la dynamique décrite jusqu'à présent peut être considérée comme le substrat dans lequel la participation féminine au marché du travail a évolué. À ce propos, l'insuffisance de données officielles assez détaillées (notamment pour les années '90) et la pénurie d'espace nous induisent à renvoyer à la partie suivante une lecture plus approfondie des caractéristiques des Marocaines; nous nous limiterons ici à citer, au niveau macro, certains indicateurs qui peuvent offrir une idée des tendances relevées dans le pays. Le premier entre eux indique que, malgré un trend mouvant - probablement dû tant à l'hétérogénéité des sources et des méthodes utilisées dans le relevé des données qu'aux fluctuations de la situation économique marocaine - le taux d'activité féminine en milieu urbain, soit $23,2 \%$ en 1992 , serait baissé à $20,9 \%$ en 2003 . L'indisponibilité de données concernant les zones rurales nous empêche d'effectuer de semblables comparaisons pour la même période. Cependant, si l'on considère seulement la période 19952003, on remarque une tendance pareille, avec des taux qui auraient décru de $39,5 \%$ à $37,3 \%$. Sur cette base, d'un côté, les taux d'activité enregistrés chez les hommes seraient respectivement $72,1 \%$ et $72,6 \%$ et, de l'autre, les taux de chômage féminin resteraient plus ou moins constants dans les villes (en passant de 25,3\% en 1992 à 25,8\% en 2003) et très bas à la campagne (en passant de 6,5\% en 1995 à 1,6\% en 2003). Mentionnons aussi que dans toutes les années pour lesquelles les informations sont disponibles, les taux d'activité (il en est de même pour les taux d'emploi) restent supérieurs en milieu rural qu'en milieu urbain; en revanche, la situation est inversée pour les niveaux de chômage. Cela pourrait être attribué à plusieurs motivations liées à la large diffusion, à la campagne, du travail informel et d'activités agricoles et artisanales, souvent pas rémunérées, exercées par les femmes notamment dans le cadre du foyer domestique; ces femmes sont fréquemment considérées dans les données officielles comme faisant partie du marché du travail. En plus, comme on le verra dans la partie 3 au niveau micro, dans ces milieux, d'après certains auteurs (CERED, 1998b), durant quelques périodes de l'année le chômage disparaîtrait en raison des multiples formes de sous-emploi extrêmement diffuses, que souvent ne sont pas considérées, par les femmes mêmes, comme de vrais «emplois», puisque elles se distinguent difficilement des fonctions de «femmes au foyer», notamment dans le cadre des «ménages complexes».

Bien évidemment, ce n'est pas dans les objectifs de ce travail d'approfondir au niveau macro et d'un point de vue économique les motivations de la dynamique tracée. Néanmoins, les pages suivantes pourront offrir d'intéressantes indications sur certaines caractéristiques individuelles des femmes qui travaillent et qui ne travaillent pas, et aussi sur les principaux déterminants socio-démographiques de la propension féminine à l'emploi, en référence aux aspects analysés jusqu'ici. 
3. Vues d'après: caractéristiques sociales, familiales et d'emploi des femmes marocaines selon une approche micro

L'objectif de cette partie est de mettre l'accent sur les principales caractéristiques de la participation des femmes au marché du travail au Maroc, à travers l'analyse et l'approfondissement dans une perspective individuelle de certains des aspects déjà examinés dans la partie précédente au niveau macro, qui s'avèrent à notre avis très discriminants.

Les jeux de données proviennent des résultats de l'Enquête Nationale sur la Population et la Santé (ENPS-II) qui a été conduite au Maroc en 1992, et de l'Enquête sur la Population et la Santé Familiale (EPSF) qui a été conduite en 2003/04 (dans la suite du texte, pour abréger nous dirons «2003»), s'inscrivant dans le programme international «Demographic and Health Surveys» (DHS). Il s'agit d'enquêtes par sondage, représentatives au niveau national et au niveau du milieu de résidence (urbain et rural), dont les échantillonnages portent respectivement sur 9.256 et 16.798 femmes âgées de 15 à 49 ans. Lors de ces enquêtes, des informations relatives à l'emploi des femmes ont été collectées.

Dans le but de distinguer les femmes qui travaillent de celles qui ne travaillent pas, nous avons considéré occupée toute femme qui au moment de l'enquête a dit avoir une activité, régulière ou non, dans le secteur formel ou informel, recevant une contrepartie en argent ou en nature. De cette façon, les femmes déclarant ne recevoir aucune rémunération en échange de leur activité ne sont pas considérées comme des femmes qui travaillent ${ }^{6}$. Bien que l'effectif de celles-ci ne soit pas très faible, notre choix a été effectué à la suite de la définition généralement reconnue de «travail», en tant qu'activité professionnelle exercée afin d'avoir une contrepartie en échange.

Selon cette définition, les femmes marocaines qui exercent une activité économique au moment de l'enquête représentent environ $16 \%$ des échantillons, tant en 1992 qu'en 2003 (Tableau 3).

La population féminine qui travaille dans les villes est plus que deux fois plus élevée qu'en milieu rural $(22,9 \%$ contre $9,5 \%$ en 1992 , et $21,5 \%$ contre $9,6 \%$ en 2003). Ce dernier résultat est apparemment en contradiction avec ce que l'on constate au niveau macro (partie 2), où les taux de travail urbains se révèlent en général plus bas que les valeurs enregistrées en milieu rural. Cependant, comme nous l'avons déjà remarqué, les données mentionnées sont à prendre avec grande précaution parce que la réalité est plus complexe qu'elle ne le paraît, la contribution féminine à l'activité économique se faisant surtout dans des circonstances de précarité, d'exploitation et de non-reconnaissance sociale et politique. En effet, le travail féminin au Maroc, notamment en milieu rural où les femmes sont particulièrement investies dans les activités domestiques et économiques à faible valeur ajoutée (agriculture, artisanat ou commerce), souffre bien souvent d'une large sous-estimation et d'une importante «invisibilité» statistique (Mejjati Alami R., 2001; Samuel O., Attané I., 2005). Le rôle des femmes dans l'économie rurale, la nature des tâches qu'elles exercent à la frontière du domestique et de l'économique, la non-prise en compte de leur pluriactivité, leur faible insertion dans le secteur formel salarié et l'excès d'emplois faiblement

${ }^{6}$ Les femmes qui travaillent sans rémunération sont 822 (soit 9,1\% du total) dans la ENPS-II de 1992 et 661 (soit $3,9 \%$ du total) dans la EPSF. 
qualifiés et faiblement rémunérés, nuisent à la reconnaissance de leur travail.

Les résultats présentés au tableau 3 mettent aussi en évidence certaines caractéristiques de l'emploi des femmes. On remarque, en premier lieu, qu'en 2003 plus des trois quarts des femmes en milieu urbain travaillaient toute l'année, contre une situation qui parait beaucoup plus précaire en milieu rural (dans $41,7 \%$ des cas, elles travaillaient de manière saisonnière).

Tableau 3 - Répartition des interviewées selon certaines caractéristiques de l'emploi par milieu de résidence, 1992 et 2003.

\begin{tabular}{|c|c|c|c|c|c|c|}
\hline \multirow{2}{*}{$\begin{array}{c}\text { Caractéristiques } \\
\text { de l'emploi }\end{array}$} & \multicolumn{3}{|c|}{1992} & \multicolumn{3}{|c|}{2003} \\
\hline & Urbain & Rural & Total & Urbain & Rural & Total \\
\hline \multicolumn{7}{|l|}{ Condition professionnelle } \\
\hline Ne travaille pas & 77,1 & 90,5 & 83,7 & 78,5 & 90,4 & 84,0 \\
\hline Travaille & 22,9 & 9,5 & 16,3 & 21,5 & 9,6 & 16,0 \\
\hline Total & 100,0 & 100,0 & 100,0 & 100,0 & 100,0 & 100,0 \\
\hline \multicolumn{7}{|l|}{ Régularité du travail ${ }^{(*)}$} \\
\hline Annuel & - & - & - & 74,5 & 36,3 & 63,9 \\
\hline Saisonnier & - & - & - & 8,8 & 41,7 & 17,9 \\
\hline Occasionnel & - & - & - & 16,7 & 22,0 & 18,2 \\
\hline Total & - & - & - & 100,0 & 100,0 & 100,0 \\
\hline \multicolumn{7}{|l|}{ Secteur d'activité } \\
\hline Agricole & 0,6 & 6,8 & 2,5 & 2,4 & 57,2 & 17,7 \\
\hline Industriel & 50,0 & 84,8 & 60,4 & 38,1 & 27,2 & 35,1 \\
\hline Tertiaire & 49,4 & 8,4 & 37,1 & 59,5 & 15,6 & 47,2 \\
\hline Total & 100,0 & 100,0 & 100,0 & 100,0 & 100,0 & 100,0 \\
\hline \multicolumn{7}{|l|}{ Statut de l'emploi } \\
\hline Dépendant & 72,9 & 27,4 & 59,2 & 79,1 & 67,0 & 75,8 \\
\hline Indépendant & 27,1 & 72,6 & 40,8 & 20,9 & 33,0 & 24,2 \\
\hline Total & 100,0 & 100,0 & 100,0 & 100,0 & 100,0 & 100,0 \\
\hline \multicolumn{7}{|l|}{ Lieu du travail } \\
\hline Au foyer & 28,5 & 65,6 & 39,6 & 20,2 & 28,3 & 22,5 \\
\hline Ailleurs & 71,5 & 34,4 & 60,4 & 79,8 & 71,7 & 77,5 \\
\hline Total & 100,0 & 100,0 & 100,0 & 100,0 & 100,0 & 100,0 \\
\hline Effectif des femmes & 4.550 & 4.706 & 9.256 & 8.997 & 7.801 & 16.798 \\
\hline
\end{tabular}

${ }^{(*)}$ Cette information n'est pas disponible pour l'année 1992

Quant aux branches d'activité et aux secteurs d'emploi, en 1992, dans la moitié des cas en milieu urbain et dans $84,8 \%$ des cas en milieu rural, les femmes travaillaient dans le secteur industriel. En revanche, en 2003, la contribution des activités agricoles, qui était secondaire lors de l'enquête précédente $(6,8 \%)$, s'établissait à 57,2\% en milieu rural. Le secteur industriel en 2003 demeurait un pôle d'attraction privilégié de l'activité féminine en milieu urbain $(38,1 \%)$, en concurrence avec le secteur tertiaire $(59,5 \%)$. En outre, on note que dans $72,9 \%$ des cas en 1992 et dans 79,1\% des cas en 2003, les femmes citadines travaillaient aux dépendances de quelqu'un d'autre. À l'opposé, dans 72,6\% des cas en 1992 et dans $33,0 \%$ des cas en 2003, les femmes travaillaient à leur propre compte. Au Maroc comme dans d'autres pays en développement, les entreprises familiales, dites aussi activités indépendantes, jouent un rôle fondamental comme source d'emploi, de façon beaucoup plus significative chez les femmes que chez les hommes. «L'emploi familial est souvent un refuge des personnes actives déscolarisées et moins qualifiées» (CERED, 1998b, page 258). En fait ces stratégies consistent à affecter les membres les moins compétitifs à l'emploi indépendant et à placer le reste dans des activités lucratives. L'auto-emploi, si marginal soit-il, est donc une forme de survie pour une large couche sociale. L'importance d'entreprises familiales, surtout dans le passé (lors de la ENPS-II), révèle que le rôle de la 
famille en tant qu'unité sociale s'étendait de la consommation à la production de biens et de services et à l'emploi, soit à l'insertion sociale des membres en âge d'activité: les ménages se portaient comme consommateurs d'emploi et/ou comme créateurs d'activité. Par contre, aujourd'hui au Maroc la famille semble être de moins en moins une base d'insertion sociale de l'individu et de son départ vers la vie active, comme le démontre l'importante raréfaction de la proportion d'emplois indépendants en milieu rural (soit 33\% contre $72,6 \%$ lors de l'enquête précédente). À ce propos, l'information sur le lieu du travail est aussi intéressant et révèle que, lors de la dernière enquête, la plupart des femmes travaillaient «ailleurs» $77,5 \%$ à l'échelle nationale), y compris les femmes qui résidaient en milieu rural (dans $71,7 \%$ des cas). Par contre, en 1992, dans 65,6\% des cas les femmes résidentes en milieu rural ayant des emplois travaillaient dans le cadre du foyer domestique.

Comme nous l'avons vu d'un point de vue macro, l'instruction est un aspect clé qui influence la participation féminine au marché du travail. Les données présentées au tableau 4 mettent en relief que, dans les deux ans observés et en milieu urbain, la proportion de «travailleuses» ayant un niveau d'éducation au moins secondaire sur le total des interviewées augmente, atteignant presque la moitié du collectif. Au contraire, les écarts entre les femmes sans aucune éducation et celles ayant un titre primaire ne semblent pas déterminer de manière significative la proportion de travailleuses. Il paraît donc que les dispositions adressées à augmenter les niveaux d'éducation féminins (partie 2) et les efforts faits dans ce sens dans le pays aient produit des résultats positifs, en augmentant la possibilité des femmes de faire partie du marché du travail, notamment pour les mieux éduquées et les «citadines». Dans les autres cas, la stagnation substantielle des valeurs observées nous fait supposer qu'il y a une contradiction entre le développement du segment instruit et formé de la force féminine de travail et la stagnation de l'emploi qui leur est disponible (CERED, 2005). Les personnes qui ont investi dans leurs études sont tentées de valoriser leurs aptitudes et leur formation dans un emploi «décent», garantissant à la fois leur sécurité financière et leur promotion sociale, et ce indépendamment de l'origine sociale. Donc, bien que les politiques d'amélioration du niveau de scolarisation des femmes aient eu des répercussions sur leur participation au marché du travail, il semble que l'on ne puisse pas parler aujourd'hui au Maroc d'un phénomène d'émancipation féminine par le travail.

Du point de vue de l'état matrimonial (Tableau 4), on constate que, en accord avec ce que l'on retrouve en littérature, la proportion des travailleuses est plus élevée chez les femmes célibataires et surtout chez les femmes divorcées, séparées ou veuves que chez les femmes mariées. Cette tendance, qui est plus significative en 2003 qu'en 1992, dépend vraisemblablement du fait que, d'une part, les femmes en rupture d'union précédente sont plus souvent obligées à travailler pour pourvoir à la subsistance de leur propre famille et, d'autre part, que les femmes célibataires, qui n'ont pas encore la tâche de soigner des enfants ${ }^{7}$, peuvent plus librement se consacrer à des activités extradomestiques (CERED, 1998b). Cependant, ce raisonnement s'applique plus aux femmes citadines qu' aux femmes rurales puisque celles-ci travaillent très souvent dans le cadre d'entreprises familiales.

À notre avis, un autre facteur s'avère décisif en ce qui concerne la participation

\footnotetext{
${ }^{7}$ La quasi-totalité des naissances au Maroc sont légitimes, c'est-à-dire qu'elles se font dans le cadre du mariage.
} 
féminine au marché du travail: la postposition de la primo nuptialité et du calendrier des naissances. Les changements importants intervenus dans le comportement nuptial et fécond des femmes marocaines, qui ont déjà été observées au niveau macro (partie 2), semblent représenter une variable discriminante de leur participation au marché du travail, surtout en milieu urbain: par exemple, en 2003, les femmes urbaines ayant une emploi se mariaient 6,1 ans plus tard que les femmes sans emploi. Cet écart est de 3,9 ans en milieu rural à la même année. Pour ce qui est de l'âge à la première naissance ${ }^{8}$, en 2003 les mères citadines qui participaient à l'activité économique avaient leur premier enfant 6,7 ans plus tard que celles qui n'y participaient pas, contre un écart de 3,2 ans en milieu rural. Cet âge s'est modifié depuis 1992, surtout en milieu rural: à cette époque, les femmes qui travaillaient ne se mariaient qu'à peine sept mois plus tard que celles sans travail et avaient leur premier enfant moins d'un an plus tard que les autres. En revanche, pour les femmes urbaines les écarts selon la condition professionnelle s'avéraient importants, soit 5,8 ans pour la primo nuptialité et 5,9 ans pour la naissance du premier enfant.

En conclusion, malgré l'état de connaissance actuel sur l'emploi demeure fragmentaire et imprégné des insuffisances conceptuelles et méthodologique qui rendent toute tentative d'analyse de la question de l'emploi au Maroc ambitieuse, ce que l'on peut remarquer est en général une substantielle concordance et homogénéité entre les informations officielles et nos données d'enquête.

Tableau 4 - Répartition des interviewées selon certaines caractéristiques socio-démographiques par milieu de résidence et condition professionnelle, 1992 et 2003.

\begin{tabular}{|c|c|c|c|c|c|c|}
\hline \multirow{2}{*}{$\begin{array}{c}\text { Caractéristiques } \\
\text { socio-démographiques }\end{array}$} & \multicolumn{3}{|c|}{ Urbain } & \multicolumn{3}{|c|}{ Rural } \\
\hline & Travaille & $\begin{array}{c}\text { Ne travaille } \\
\text { pas }\end{array}$ & Total & Travaille & $\begin{array}{c}\text { Ne travaille } \\
\text { pas }\end{array}$ & Total \\
\hline \multicolumn{7}{|c|}{1992} \\
\hline \multicolumn{7}{|l|}{ Niveau d'instruction } \\
\hline Aucune & 19,6 & 80,4 & 100,0 & 9,3 & 90,7 & 100,0 \\
\hline Primaire & 22,7 & 77,3 & 100,0 & 10,3 & 89,7 & 100,0 \\
\hline Secondaire ou plus & 42,2 & 57,8 & 100,0 & 50,0 & 50,0 & 100,0 \\
\hline \multicolumn{7}{|l|}{ État matrimonial } \\
\hline Célibataire & 26,5 & 73,5 & 100,0 & 9,9 & 90,1 & 100,0 \\
\hline Mariée & 17,9 & 82,1 & 100,0 & 8,6 & 91,4 & 100,0 \\
\hline Veuve/Divorcée/Séparée & 38,5 & 61,5 & 100,0 & 18,7 & 81,3 & 100,0 \\
\hline$\hat{A}$ ge médian au premier mariage $e^{(*)}$ & 27,4 & 21,6 & 23,0 & 20,5 & 20,0 & 20,1 \\
\hline Âge médian à la $1^{r e}$ naissance ${ }^{(*)}$ & 29,2 & 23,3 & 24,8 & 22,5 & 21,8 & 22,2 \\
\hline \multicolumn{7}{|c|}{2003} \\
\hline \multicolumn{7}{|l|}{ Niveau d'instruction } \\
\hline Aucune & 20,2 & 79,8 & 100,0 & 10,0 & 90,0 & 100,0 \\
\hline Primaire & 18,7 & 81,3 & 100,0 & 8,6 & 91,4 & 100,0 \\
\hline Secondaire ou plus & 46,9 & 53,1 & 100,0 & 45,9 & 54,1 & 100,0 \\
\hline \multicolumn{7}{|l|}{ État matrimonial } \\
\hline Célibataire & 25,8 & 74,2 & 100,0 & 13,0 & 87,0 & 100,0 \\
\hline Mariée & 17,7 & 82,3 & 100,0 & 7,0 & 93,0 & 100,0 \\
\hline Veuve/Divorcée/Séparée & 41,5 & 58,5 & 100,0 & 25,5 & 74,5 & 100,0 \\
\hline$\hat{A}$ ge médian au premier mariage $e^{(*)}$ & 28,9 & 22,8 & 24,3 & 24,9 & 21,0 & 21,3 \\
\hline$\hat{A}$ ge médian à la $1^{r e}$ naissance ${ }^{(*)}$ & 31,2 & 24,5 & 26,3 & 26,0 & 22,8 & 23,0 \\
\hline
\end{tabular}

\footnotetext{
${ }^{8}$ Nous précisons que dans cette partie nous n'avons pas mentionné les valeurs de l'ISF issues des enquêtes ENPSII et EPSF, puisque elles ont déjà été indiquées dans la partie 2 (Tableau 1).
} 


\section{Quelques déterminants de l'emploi féminin: un modèle interprétatif}

On a effectué l'analyse de la participation des Marocaines au marché du travail en utilisant deux types de régression: logistique et multinomiale. Dans la première, la variable dépendante assume la valeur 1 si l'interviewée a un emploi rémunéré, et 0 autrement (partie 3). Dans le modèle multinomiale, où les femmes sans emploi ont été exclues, nous avons divisé les interviewées selon les trois secteurs d'emploi (agricole, industriel, tertiaire), en considérant le secteur industriel comme la modalité de référence. Les analyses paramétriques effectuées incluent un ensemble de variables indépendantes, certaines continues et d'autres dichotomiques, qui peuvent être classifiées en trois groupes différents (individuel, familial et social), conformément à certains milieux d'intérêt mentionnés en littérature (partie 2).

En ce qui concerne les caractéristiques individuelles, deux variables continues ont été insérées dans les modèles: l'âge au moment de l'interview ${ }^{9}$, pour laquelle nous supposons une relation à «U retourné» avec la variable dépendante, et le nombre d'ans d'éducation qui, au contraire, serait en relation positive avec la propension des Marocaines à travailler.

Dans le cadre du contexte familial, nous avons considéré trois aspects: le premier, exprimé par une variable dichotomique, concerne l'état matrimonial de l'interviewée; le deuxième, exprimé par une variable continue, concerne le nombre de membres du ménage qui cohabitent (y compris l'interviewée); le dernier, exprimé à travers trois variables dichotomiques, concerne le type de relation entre l'interviewée et le chef de ménage ${ }^{10}$. À travers ces variables nous souhaitons analyser l'influence des caractéristiques des ménages sur la participation féminine au marché du travail. Notre hypothèse est qu'un modèle familial plus «traditionnel» (caractérisé par interviewées mariées, un chef de ménage de sexe masculin et par un nombre de membres plus élevé) réduirait le travail des femmes. Au contraire, des modèles familiaux «différents» (ménages dirigés par des femmes, interviewées célibataires, taille des ménages réduite) seraient des aspects favorisant l'emploi féminin.

Finalement, le contexte social comprend trois variables dichotomiques: le milieu de résidence (urbain vs rural); le niveau d'éducation du conjoint (secondaire et plus vs aucune/primaire) et son secteur d'activité (secteur agricole vs autre). Les analyses et les considérations effectuées dans les parties précédentes ont déjà mis en relief une nette contraposition de la condition féminine par milieu de résidence. On en dérive l'hypothèse que les profondes transformations de la société marocaine suivent deux vitesses: plus rapides et orientées vers un marché du travail plus dynamique en milieu urbain; plus lentes et orientées vers le maintien de comportements traditionnels en milieu rural. En tel sens, aussi la variable concernant l'éducation du mari exprimerait une condition orientée vers des comportements flexibles et plus favorables à une participation active de la femme dans le marché du travail. En revanche, un conjoint qui travaille dans le secteur agricole favoriserait le travail de la femme dans le même genre d'activité.

Les résultats de la régression logistique sont présentés au tableau 5. En

\footnotetext{
${ }^{9}$ Pour cette variable nous avons aussi inséré son terme quadratique.

${ }^{10}$ En particulier, quatre modalités ont été dichotomisées (interviewée est chef de ménage, femme du chef de ménage, fille du chef de ménage, autre type de relation avec le chef de ménage) dont la première est prise comme référence.
} 
particulier, nous avons estimé un premier modèle où les données des deux enquêtes sont utilisées de façon conjointe. Seules les caractéristiques individuelles et le milieu de résidence ont été insérées comme variables indépendantes et sont significatives. La variable la plus intéressante est sûrement celle qui exprime l'an où l'interviewée a participé à l'enquête (variable dichotomique EPSF 2003). Toute autre condition étant pareille, pour les femmes interviewées en 2003 la propension à participer au marché du travail se réduit significativement $(\mathrm{p}<0,01)$, soit d'environ 60\% ( $\beta$ : -0,92) en comparaison avec les interviewées en 1992. Ce résultat est cohérent avec ce que nous avons mis en évidence dans les parties précédentes.

Le deuxième et le troisième modèles mettent l'accent sur les principaux déterminants de la participation féminine au marché du travail dans une optique comparative entre les deux enquêtes. En ce qui concerne le contexte individuel, les résultats confirment nos hypothèses précédentes pour 1992 et 2003. Notamment on remarque que l'augmentation des années d'éducation cause une augmentation significative du travail féminin, soit environ $8 \%$ et $6 \%$ (respectivement en 1992 et en 2003). Confirmant ce que nous avons mis en relief dans les parties 2 et 3, les plans d'action entrepris au Maroc dans le but de promouvoir la scolarisation féminine favoriseraient l'accès des femmes aux activités productives. Quant aux variables concernant le contexte familial, tous les coefficients assument une valeur négative. En particulier, le fait d'être mariée réduit beaucoup l'emploi féminin ( $\beta$ : -1,167 dans la ENPS de 1992 et -1,507 dans la EPSF de 2003); nous avons le même effet dans tous les cas où ce n'est pas l'interviewée qui dirige le ménage. En synthèse, on peut noter que, dans les dix dernières années, la figure du chef de ménage assume un rôle primaire pour la subsistance du noyau familial et que la condition de la femme au Maroc n'a pas beaucoup changé, puisque sa participation au marché du travail, surtout dans les ménages traditionnels, demeure défavorisée. Finalement, en ce qui concerne le contexte social, tous les modèles estimés révèlent une propension plus élevée à un emploi rémunéré pour les femmes qui résident en ville ( $\beta$ : 0,513 en 1992 et 0,425 en 2003). Dans ces milieux, où il $\mathrm{y}$ aurait aussi une forte concentration de femmes ayant des emplois plus «formels» et rémunérés ${ }^{11}$, on retrouverait des comportements moins traditionnels et plus orientés vers la logique du marché de travail. L'activité féminine semble aussi être favorisée par la présence d'un conjoint ayant un niveau d'éducation moyen/élevé ( $\beta$ : 0,276 dans la ENPS de 1992 et 0,485 dans la EPSF de 2003). La présence d'un mari qui travaille dans le secteur agricole produit un effet semblable. Cette circonstance, qui laisse présumer la présence des femmes dans les activités professionnelles exercées dans le cadre familial, surtout en agriculture, est encore plus évidente dans les résultats du modèle où nous analysons les principaux déterminants de la participation des interviewées dans les trois secteurs d'activité (Tableau 6). Dans ces analyses, les deux enquêtes ont été considérées conjointement afin de produire plus d'observations.

\footnotetext{
${ }^{11}$ Relativement à cette variable, il est intéressant de remarquer que, utilisant comme variable dépendante la présence dans le marché du travail de femmes pas rémunérées, les résultats obtenus sont le contraire. Dans d'autres termes, si l'on considère aussi le travail sans rémunération, les femmes résidentes en milieu urbain présentent une propension plus basse à l'occupation ( $\beta$ : - 0,130 dans la ENPS de 1992 et $-0,101$ dans la EPSF de 2003). Cela renforcerait l'opinion (parties 2 et 3 ) selon laquelle en milieu rural le travail informel des femmes est largement diffusé et expliquerait pourquoi, selon les statistiques officielles, les taux d'activité féminine sont plus élevés en milieu rural qu'en milieu urbain.
} 
Tableau 5 - Participation féminine au marché du travail, régression logistique.

\begin{tabular}{|c|c|c|c|}
\hline \multirow{2}{*}{ Variables } & ENPS 1992 + EPSF 2003 & ENPS 1992 & EPSF 2003 \\
\hline & Coeff. (St.Er.) Sig. & Coeff. (St.Er.) Sig. & Coeff. (St.Er.) Sig \\
\hline Constante & $-5,016(0,191) * * *$ & $-4,532(0,367) * * *$ & $-4,847(0,268) * * *$ \\
\hline \multicolumn{3}{|c|}{ An de l'enquête (réf: ENPS 1992) } & \\
\hline Âge à l'interview & $0,163(0,012) * * *$ & $0,246(0,024) * * *$ & $0,254(0,017) * * *$ \\
\hline Âge à l’interview ^2 & $-0,002(0,001) * * *$ & $-0,003(0,001) * * *$ & $-0,003(0,001) * * *$ \\
\hline Années d'éducation & $0,077(0,004) * * *$ & $0,076(0,008) * * *$ & $0,058(0,005) * * *$ \\
\hline \multicolumn{4}{|c|}{ État matrimonial (réf: interviewée célibataire) } \\
\hline Mariée & & $-1,167(0,121) * * *$ & $-1,507(0,094) * * *$ \\
\hline Taille du ménage & & $-0,117(0,010)$ & $-0,043(0,009) * * *$ \\
\hline $\begin{array}{l}\text { Type de lien avec le } \\
\text { Femme } \\
\text { Fille } \\
\text { Autre }\end{array}$ & ef de ménage (réf: interview & $\begin{array}{l}\text { le ménage) } \\
-0,812(0,133) * * * \\
-1,247(0,126) * * * \\
-1,080(0,133) * * *\end{array}$ & $\begin{array}{l}-0,591(0,097) * * * \\
-0,947(0,089) * * * \\
-1,045(0,097) * * *\end{array}$ \\
\hline \multicolumn{4}{|c|}{ Milieu de résidence (réf: milieu rural) } \\
\hline $\begin{array}{l}\text { Niveau d'éducation } \\
\text { Secondaire ou plus }\end{array}$ & conjoint (réf: aucune et pri & $0,276(0,086) * * *$ & $0,485(0,069) * * *$ \\
\hline $\begin{array}{l}\text { Secteur d'activité du } \\
\text { Secteur agricole }\end{array}$ & njoint (réf: industriel et ter & $0,456(0,367) * *$ & $0,059(0,096) * * *$ \\
\hline $\begin{array}{l}\text { Interviewées } \\
\mathrm{R}^{2}\end{array}$ & $\begin{array}{r}25.901 \\
0,062\end{array}$ & $\begin{array}{l}9.166 \\
0,103\end{array}$ & $\begin{array}{r}16.735 \\
0,119\end{array}$ \\
\hline
\end{tabular}

$* \mathrm{p}<0,10 ; * * \mathrm{p}<0,05 ; * * * \mathrm{p}<0,01$.

Tableau 6 - Participation féminine au marché du travail, régression multinomiale.

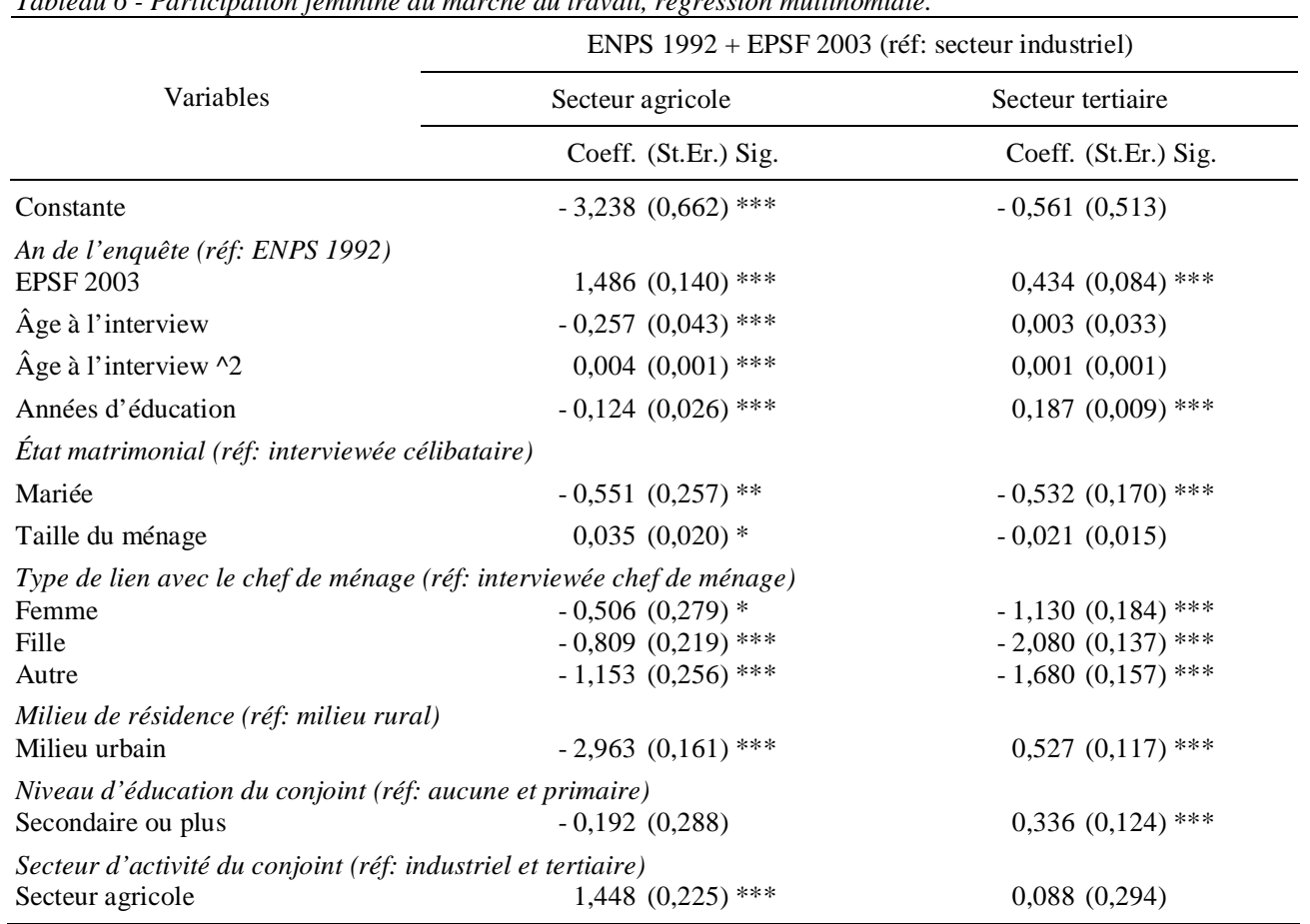

Interviewées: $4.310 ; \mathrm{R}^{2}: 0,333$

$* \mathrm{p}<0,10 ; * * \mathrm{p}<0,05 ; * * * \mathrm{p}<0,01$. 
Le travail dans les secteurs agricole $(\beta: 1,486)$ et tertiaire $(\beta: 0,434)$ serait favorisé en comparaison avec le secteur industriel en 2003, comme on l'a déjà dit dans la partie 3. De plus, on remarque que l'augmentation des années d'étude ( $\beta$ : $0,124)$, la résidence urbaine $(\beta:-2,963)$ et un conjoint ayant un niveau d'éducation au moins secondaire $(\beta$ : - 0,192$)$ réduisent l'activité féminine dans le secteur agricole. Les coefficients de ces variables assument des valeurs positives si l'on considère le secteur tertiaire. Il est intéressant que le signe négatif du coefficient qui correspond à l'âge au moment de l'enquête $(\beta$ : $-0,257)$ dans le modèle concernant le secteur agricole. L'apport des bras féminins dans ce secteur d'emploi serait donc exploité surtout pendant les premiers âges.

En conclusion, les analyses effectuées mettraient en évidence l'existence au Maroc d'un marché du travail féminin en évolution. À plusieurs égards, ce marché reste lié, surtout en milieu rural, à des modèles familiaux traditionnels dans lesquels le rôle des femmes est limité à des fonctions secondaires, souvent en dehors du travail rémunéré.

\section{Considérations conclusives}

Le scénario qui a été tracé jusqu'à présent fournit des indications problématiques sur la dynamique qui caractérise la participation féminine au marché du travail marocain dans la période considérée, et sur les relations qui existent entre ce phénomène et les conditions socio-démographiques des femmes.

D'un côté, on ne peut pas nier que des facteurs tels que l'instruction et les caractéristiques familiales jouent un rôle important dans la détermination de la main-d'œuvre féminine. De l'autre côté, le processus général de «modernisation» du pays ne semble pas avoir mené, comme dans d'autres pays, à un plus grand désir chez les femmes de participer aux activités productives. Cependant, il faudrait évaluer cette constatation, issue de façon complémentaire des informations officielles et des données d'enquête, à la lumière de paradigmes qui ne se refont pas aux schémas interprétatifs «occidentaux», d'après lesquels une augmentation de la proportion de femmes qui travaillent est un indicateur d'une augmentation du niveau de développement dans le pays.

À ce propos, il faut reconnaître que même la définition du mot «travail» se prête à plusieurs interprétations. En effet, comme nous l'avons déjà remarqué, la littérature marocaine met en évidence que, surtout en milieu rural, la frontière entre les fonctions remplies par les femmes dans le cadre du foyer domestique et les activités productives au sens strict est très faible. En outre, les résultats laissent entrevoir que, à exception d'une faible proportion de cas principalement concentrés dans les zones urbaines et dans le secteur tertiaire, les emplois exercés par les nordafricaines observées sont marqués par une basse qualification et par un haut niveau de précarité et de marginalité. Cela semble lié plus à la nécessité de repérer ou d'augmenter les ressources économiques - parfois insuffisantes - indispensables à la subsistance du noyau familial, qu'au désir d'atteindre un niveau de réalisation professionnelle et d'émancipation satisfaisantes.

$\mathrm{Au}$ lieu de conclure ce travail par des considérations indiscutables, il nous paraît plus opportun de nous poser une question: la stabilité substantielle de la participation féminine au marché du travail au Maroc peut-elle vraiment être vue 
comme une «occasion perdue» dans le chemin d'une amélioration du statut des femmes? Ou plutôt pourrait-elle être considérée comme une étape obligée du processus de leur affranchissement des activités dégradantes d'un système de production arriéré, étape nécessaire afin d'atteindre un niveau de développement global plus élevé qui tient en compte les caractéristiques démographiques, économiques, sociales et culturelles propres à ce pays maghrébin?

\section{Remerciements}

Ce travail a été présenté au XIVème Colloque de l'Association Internationale des Démographes de Langue Française (AIDELF), qui a eu lieu à Aveiro du 18 au 22 Septembre 2006. Les auteurs tiennent à remercier le Max Planck Institute for Demographic Research (Rostock) pour le précieux support financier, Agnès Adjamagbo et Laura Bernardi pour les commentaires fructueux et Diane Monique Harris pour la révision du français. 


\section{Références bibliographiques}

Azelmat M., AYAD M., Housni E.A., 1993. «Enquête Nationale sur la Population et la Santé (ENPS-II) 1993», Ministère de la Santé Publique (Maroc), Macro International Inc.

Azelmat M., Ayad M., Abdelmoneim A., 2005. «Enquête sur la Population et la Santé Familiale (EPSF) 2003/04», Ministère de la Santé Publique (Maroc), Macro International Inc, Ligue des États Arabes.

BoutATA M., 2005. «La relation emploi-formation au Maroc: des ajustements actuels et perspectives d'une meilleure articulation», in CERED, Situation socioéconomique et défis démographique au Maroc, Rabat.

CERED, 1997. «Situation et perspectives démographiques du Maroc», Rabat.

CERED, 1998a. «Genre et éducation», in CERED, Genre et développement, Rabat.

CERED, 1998b. «Famille au Maroc», Rabat.

CERED, 2005. «Situation socio-économique et défis démographique au Maroc»,

Rabat.

CERED, années variées, http://www.cered.hcp.ma/.

ChaOuAi A., 1998. «Promotion des femmes et condition de la femme», in: CERED, Population \& Développement au Maroc, Rabat.

D'ADDATO A.V., 2006a. «In tema di transizione della fecondità: comportamenti familiari dei marocchini in patria e in Italia», Thèse doctorale, Université de Bari.

D'ADDATO A.V., 2006b. «Progression to third birth in Morocco in the context of fertility transition », Demographic Research, 15, n. 19, http://www.demographicresearch.org/Volumes/Vol15/19/15-19.pdf.

DE SARno-Prignano A., El MouaAtamid B., PATERno A., 2003. «Mobilità interna, migrazioni internazionali ed implicazioni demografiche e sociali in Marocco», in Natale M., Moretti E. (sous la direction de), Siamo pochi o siamo troppi? Alcuni aspetti delle relazioni tra evoluzione demografica e sviluppo economico e sociale, Franco Angeli, Milano.

DIRECTION DE LA STATISTIQUE, années variées, http://www.statistic-hcp.ma/.

HANCOCK P., 2006. «Women, work and empowerment: a portrait of women workers in two of Sri Lanka's Export Processing Zones», Norwegian Journal of Geography, 60, n. 3.

HARRAMI N., 2000. «La condition féminine au Maroc: Éléments d'appréciation», in CERED, "Situation socio-économique et défis démographique au Maroc», Rabat.

HDDIGUI E.M., 1998. «Éducation: Stratégie nationale en matière de scolarisation et d'alphabétisation», in CERED, Population \& Développement au Maroc, Rabat.

MEJJATI AlAMI R., 2001. «Femmes et marché du travail au Maroc», Communication présentée au XXIV ${ }^{\mathrm{e}}$ Congrès Général de la Population (UIESP), Salvador, Brésil.

MosLEH K., 2000. «La condition juridique de la femme au Maroc? Cinq ans après la Conférence Internationale du Caire sur la Population et le Développement», in CERED, Situation socio-économique et défis démographiques au Maroc, Rabat.

SAMUEl O., ATTANÉ I. (sous la direction de), 2005. «Femmes, famille, fécondité: De la baisse de la fécondité à l'évolution du statut des femmes», Revue du Tiers Monde, XLVI, n. 182. 